\title{
TRATAMENTO CIRÚRgICO POSTERGADO DA PANCREATITE AGUDA BILIAR
}

\author{
SURGICAL TREATMENT OF ACUTE BILIARY PANCREATITIS
}

RESUMO: A análise do tratamento cirúrgico de doentes portadores de pancreatite biliar mostra a existência de controvérsias em relação à oportunidade da intervenção, principalmente se deve ser precoce ou postergada. Do mesmo modo, a possibilidade do emprego de procedimentos endoscópicos no pré, intra ou pós-operatório e o advento da videolaparoscopia, trouxeram novos aspectos à discussão. Não existe consenso sobre a escolha da melhor conduta. Em função disso, analisamos retrospectivamente os resultados imediatos de 107 doentes portadores de forma leve de pancreatite, todos com menos de três sinais de gravidade, segundo o critério de estratificação proposto por Ranson, e que foram submetidos ao tratamento cirúrgico postergado na mesma internação, no período de janeiro de 1988 a maio de 1999, tanto por via convencional como por via laparoscópica. Desses, 80 doentes (75\%) eram do sexo feminino, $90 \%$ da raça branca e a média de idade foi de 46 anos. Os doentes foram operados em média após 9,5 dias de internação e receberam alta hospitalar após 2,9 dias, o que resultou numa permanência hospitalar média de 12,6 dias. A colangiografia intra-operatória foi realizada em 102 casos (96\%) e a colangiografia endoscópica préoperatória em 24 doentes $(22,4 \%)$. Os resultados mostraram incidência de coledocolitíase em 25 casos (23\%), taxa de morbidade de $12 \%$ e mortalidade nula. Dos 107 casos estudados, $64(60 \%)$ foram operados pela via de acesso convencional e 43 (40\%) pela via laparoscópica. A comparação dos resultados entre as vias de acesso empregadas mostrou diferença estatística significante em relação ao intervalo de tempo pós-operatório, que foi menor nos doentes submetidos à via de acesso laparoscópica. Concluímos, assim, que o tratamento cirúrgico postergado de doentes portadores de pancreatite biliar na forma leve apresenta baixas morbidade e mortalidade e pode ser feito tanto pela via convencional como pela via laparoscópica. A presença de coledocolitíase, nesta casuística, não contribuiu para aumentar os índices de complicações pós-operatórias e nem a mortalidade.

Unitermos: Pancreatite aguda, Pancreatite biliar, Tratamento cirúrgico, Colecistectomia videolaparoscópica.

\section{INTRODUÇÃO}

O tratamento da pancreatite aguda biliar sempre gerou controvérsias. Se no início do século discutiu-se a indicação do tratamento cirúrgico, a partir dos anos 50 passou-se a pensar na melhor oportunidade da interven- ção. Na década de 70, a utilização dos procedimentos endoscópicos modificou a abordagem terapêutica inicial e, mais recentemente, o desenvolvimento da videocirurgia também repercutiu no tratamento. ${ }^{1-9}$

A padronização da colecistectomia laparoscópica tornou-a a via de acesso preferencial para o tratamento

1. Professor Assistente - Doutor do Departamento de Cirurgia da FCMSCSP

2. Residente de Cirurgia do Departamento de Cirurgia da FCMSCSP

3. Pós-Graduando do Departamento de Cirurgia da FCMSCSP

4. Professor Adjunto - Doutor Chefe do Grupo de Via Biliar e Pâncreas do Departamento de Cirurgia da FCMSCSP

5. Professor Titular do Departamento de Cirurgia da FCMSCSP

6. Professor Titular - Chefe do Serviço de Emergência do Departamento Cirurgia da FCMSCSP

Recebido em 21/9/99

Aceito para publicação e 14/3/2000

Trabalho realizado no Departamento de Cirurgia da Faculdade de Ciências Médicas da Santa Casa de São Paulo — FCMSCSP. 
cirúrgico da litíase vesicular. No entanto, as particularidades do doente portador de pancreatite biliar levam a algumas reflexões em relação ao seu emprego no tratamento dessa afecção.

Assim, ao contrário de litíase vesicular não complicada, em que, na maioria das vezes, a intervenção se limita à colecistectomia com ou sem colangiografia intra-operatória, na pancreatite biliar pode ser necessária a manipulação da via biliar para a retirada de cálculos ou, ainda, a associação de procedimentos cirúrgicos e endoscópicos.

Embora o tratamento cirúrgico postergado, realizado na mesma internação, após a melhora dos sintomas da pancreatite, ainda seja a conduta preferencial para os doentes portadores da forma leve da afecção, persistem muitas dúvidas em relação à necessidade da realização de procedimentos endoscópicos pré-operatórios e à escolha da via de acesso para a intervenção cirúrgica.

Se a escolha é pela via de acesso laparoscópica associada à papilotomia endoscópica pré-operatória, deve-se levar em conta a possibilidade de ocorrência de complicações nos dois procedimentos. Por outro lado, se a opção é pela videocirurgia e, no intra-operatório, constata-se a presença de cálculo no colédoco, fica a dúvida se é melhor tentar a sua remoção laparoscópica, tratar a coledocolitíase por via endoscópica no pós-operatório ou converter a cirurgia para a via aberta.

Em vista disso, o presente estudo teve o objetivo de analisar os resultados imediatos do tratamento cirúrgico postergado de doentes portadores de forma leve de pancreatite biliar, operados pela via de acesso convencional ou laparoscópica.

\section{PACIENTES E MÉTODOS}

Analisamos retrospectivamente 107 doentes portadores de pancreatite aguda biliar submetidos a tratamento operatório no Departamento de Cirurgia da Faculdade de Ciências Médicas da Santa Casa de São Paulo, no período de janeiro de 1988 a maio de 1999. Desses, 80 doentes eram do sexo feminino $(74,7 \%)$ e a média de idade de 46,6 anos.
Foram incluídos no presente estudo somente os doentes com diagnóstico de pancreatite aguda biliar baseados em dados clínicos, laboratoriais e ultra-sonográficos. Todos eles foram classificados, pelos critérios de estratificação de Ranson ${ }^{3}$, como portadores de forma leve de pancreatite, por apresentarem menos de três sinais de gravidade. Todos responderam satisfatoriamente ao tratamento clínico inicial, sendo submetidos ao tratamento cirúrgico postergado na mesma internação.

Dividimos os doentes em dois grupos (1 e 2), segundo a via de acesso empregada, e analisamos os seguintes parâmetros: icterícia na admissão; dilatação da via biliar à ultra-sonografia pré-operatória; tempo de história ( intervalo de tempo entre o aparecimento dos sintomas e a internação) e tempo de pré-operatório ( intervalo de tempo entre a internação e a operação).

Estudamos as seguintes variáveis para analisarmos os resultados do tratamento operatório postergado e para compararmos os grupos segundo as vias de acesso utilizadas: tempo de pós-operatório ( intervalo de tempo entre a operação e a alta hospitalar); tempo de permanência hospitalar; procedimentos endoscópicos e cirúrgicos realizados; complicações e mortalidade.

$\mathrm{Na}$ análise estatística dos resultados utilizamos os testes do qui-quadrado e o teste exato de Fisher para comparação das proporções em relação à presença ou não das variáveis.

\section{RESULTADOS}

Verificamos que, dos 107 doentes estudados, 59 não relataram episódios prévios de icterícia ou pancreatite. No entanto, $50(46,7 \%)$ apresentavam-se ictéricos na admissão (Tabela 1).

Os achados ultra-sonográficos pré-operatórios revelaram que 47 doentes $(43,9 \%)$ apresentavam dilatação da via biliar (Tabela 2).

Em relação aos intervalos de tempo estudados constatamos que os doentes procuraram o hospital com um quadro doloroso de 3,1 dias e foram operados com cerca

Tabela 1

Dados clínicos, períodos estudados, morbidade e mortalidade em portadores de pancreatite biliar (jan/98 - mai/99)

\begin{tabular}{|c|c|c|c|c|}
\hline & \multicolumn{2}{|c|}{ Convencional } & \multicolumn{2}{|c|}{ Laparoscópico } \\
\hline & $\begin{array}{l}N \\
64\end{array}$ & $\begin{array}{c}\% \\
59,8\end{array}$ & $\begin{array}{l}N \\
43\end{array}$ & $\begin{array}{c}\% \\
40,2\end{array}$ \\
\hline Com icterícia & 30 & 46,9 & 20 & 46,5 \\
\hline Sem icterícia & 34 & 53,1 & 23 & 53,5 \\
\hline Tempo de história (em dias) & 2,9 & & 3,4 & \\
\hline Tempo de pré-operatório & 9,4 & & 9,8 & \\
\hline Tempo de pós-operatório & 3,6 & & 1,9 & \\
\hline Permanência hospitalar & 13,1 & & 11,9 & \\
\hline Morbidade & & 14,1 & & 9,3 \\
\hline Mortalidade & 0 & & 0 & \\
\hline
\end{tabular}


Tabela 2

Dados ultra-sonográficos em portadores de pancreatite biliar

\begin{tabular}{l|cc|cc}
\hline & \multicolumn{2}{|c|}{ Convencional } & \multicolumn{2}{c}{ Laparoscópico } \\
\cline { 2 - 5 } & $N$ & $\%$ & $N$ & 43 \\
\hline Microlitíase & 64 & 59,8 & 30 & 40,2 \\
Dilatação das vias biliares & 35 & 54,7 & 22 & 69,8 \\
Alterações pancreáticas & 25 & 39,1 & 9 & 51,2 \\
\hline
\end{tabular}

de 9,5 dias de evolução. O período pós-operatório foi de 2,9 dias e a permanência hospitalar de 12,6 dias. Verificamos que a comparação dos resultados entre os dois grupos mostrou diferença estatística significante em relação ao tempo de pós-operatório. Os doentes submetidos à colecistectomia videolaparoscópica apresentaram menor tempo de pós-operatório (Tabela 1).

Dos 107 doentes estudados, em 82 (76,6\%), constatou-se apenas colelitíase e em $25(23,4 \%)$ verificou-se coledocolitíase associada. Dos portadores de coledocolitíase, 19 foram tratados pela via convencional e seis pela via laparoscópica. Realizamos 24 colangiopancreatografias endoscópicas retrógadas no pré-operatório, das quais em $14(56 \%)$ não foram constatados cálculos no colédoco e em dez (34\%) detectou-se cálculo na via biliar. Em um caso o exame endoscópico não constatou cálculo no colédoco mas evidenciou divertículo justapapilar. Dos dez casos de coledocolitíase associada, em sete o cálculo foi retirado após papilotomia endoscópica. Em dois o procedimento não teve sucesso (um foi submetido posteriormente à cirurgia convencional e um à cirurgia laparoscópica). Dos sete casos cujo procedimento endoscópico foi bem-sucedido, quatro foram posteriormente operados pela via laparoscópica e três pela via convencional (Figura 1).

Dos 64 doentes submetidos à cirurgia convencional, dos quais 19 apresentavam coledocolitíase associada, em três o cálculo foi retirado por via endoscópica, em outros três houve a necessidade de se associar à colecistectomia à papilotomia transduodenal e em um o cálculo conseguiu ser retirado por via transcística. Nos 12 restantes, associou-se a colecistectomia à coledocotomia com retira- da do cálculo e drenagem à Kehr. Em um caso, que apresentava divertículo justapapilar, sem cálculo no colédoco, realizou-se também a derivação biliodigestiva coledocoduodenal (Figura 2).

Dos 43 doentes submetidos à cirurgia laparoscópica, dos quais seis apresentavam coledocolitíase associada, em quatro o cálculo foi retirado por via endoscópica, em um por coledocotomia, após conversão do procedimento (submetido anteriormente à papilotomia endoscópica sem sucesso), e em um por via transcística (Figura 2).

Constatamos que 13 doentes $(12,1 \%)$ apresentaram complicações pós-operatórias (Figura 3), não sendo observada mortalidade nesta casuística.

\section{DISCUSSÃO}

Um dos objetivos do tratamento cirúrgico da pancreatite biliar é eliminar a doença calculosa vesicular através da colecistectomia, visando a impedir a recidiva dos surtos pela migração de novos cálculos. A intervenção pode ser realizada na fase precoce da evolução da doença, na fase postergada ( na mesma internação, mas após a remissão dos sintomas ), ou na fase tardia, em internação posterior.

A elevada incidência da recidiva de surto de pancreatite observada após seis meses do surto inicial, mostrou que o tratamento na fase tardia não era o ideal e, por isso, passou a ser o menos empregado. Desse modo, a principal controvérsia em relação à oportunidade da intervenção tem sido se ela deve ser precoce ou postergada.

Apesar de vários autores já terem estudado os resultados dessas duas opções de intervenção, notando-se até

Figura 1 - Procedimentos endoscópicos realizados no pré-operatório (24 doentes)

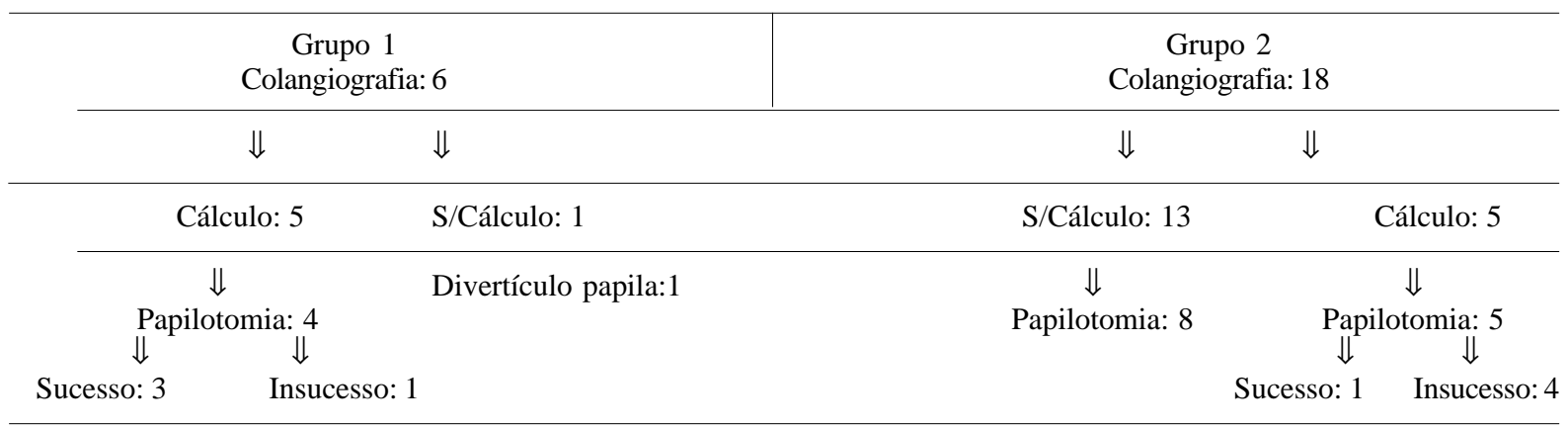


Figura 2 - Procedimentos cirúrgicos e endoscópicos realizados em 107 doentes portadores de pancreatite biliar (jan/98 - mai/99)

\begin{tabular}{|c|c|}
\hline Grupo 1 & Grupo 2 \\
\hline • Colecistectomia convencional: 64 & - Colecistectomia laparoscópica: 43 \\
\hline - Coledocotomia + Kehr: 12 (18.8\%) & • Conversão: $6(14.0 \%)$ \\
\hline - Papilotomia transduodenal: $3(4.7 \%)$ & - Coledocotomia: $1(2.3 \%)^{* *}$ \\
\hline - Colédoco-duodenal: $1(1.6 \%)^{*}$ & $\begin{array}{l}\text { - Exploração transcística: } 1(2.3 \%) \\
\text { - Exploração transcística: } 1(1.6 \%)\end{array}$ \\
\hline
\end{tabular}

*Doente portador de divertículo justapapilar

**Doente submetido à papilotomia endoscópica pré-op. e conversão do procedimento laparoscópico.

Figura 3 - Complicações pós-operatórias

\begin{tabular}{|c|c|c|c|}
\hline & Grupo1 & & Grupo 2 \\
\hline $\begin{array}{r}9 \text { casos } \\
(14.1 \%)\end{array}$ & $\begin{array}{ll}\text { Infecção de parede } & : 5 \\
\text { Fístula biliar } & : 1 \\
\text { Abscesso intracavitário } & : 1 \\
\text { Evisceração } & : 1 \\
\text { Hematoma de parede } & : 1\end{array}$ & $\begin{array}{l}4 \text { casos } \\
(9.3 \%)\end{array}$ & $\begin{array}{l}\text { Coleção intracavitária : } 1 \\
\text { Infecção de parede }\end{array}$ \\
\hline
\end{tabular}

uma maior tendência na indicação do tratamento postergado, um dos aspectos que sempre chamou a atenção é que nem sempre se comparam resultados entre doentes com a mesma gravidade da pancreatite. Muitas vezes são analisados doentes portadores de necrose pancreática com portadores de pancreatite edematosa, explicando-se, assim, a diversidade dos resultados (Burch et al). ${ }^{9,10,11}$

Em função disso, ao idealizarmos este trabalho, nos interessou comparar os resultados da cirurgia postergada em portadores de pancreatite biliar leve, uma vez que este grupo representa a maioria dos casos. A seleção dos doentes, embora feita através de critérios de estratificação de gravidade, geralmente reflete aqueles casos que apresentam melhora do quadro doloroso em dois a quatro dias de internação, normalizam os níveis de amilase sérica e conseguem ser realimentados.

Acreditando que a etiopatogenia da pancreatite biliar está relacionada à obstrução transitória da papila pela passagem ou impactação de um cálculo e que, na maioria das vezes, o cálculo migra espontaneamente para o duodeno, passamos a empregar o tratamento cirúrgico postergado com o objetivo que a operação se limitasse à colecistectomia com a colangiografia intra-operatória. Basicamente, a exemplo de autores que empregaram esse tipo de intervenção, buscávamos alcançar baixos índices de morbidade e mortalidade e menor incidência de coledocolitíase. ${ }^{12,13}$

Nossos resultados mostraram uma incidência de coledocolitíase de 23,4\% (25 doentes), morbidade de 12,0\% (13 casos) e mortalidade nula, o que está de acordo com os resultados da literatura. Com o emprego da via de acesso laparoscópica pretendíamos verificar a extensão dos benefícios da cirurgia minimamente invasiva nesta situação.

O grande problema no emprego dessa via de acesso para a realização da cirurgia postergada é que o tratamento de eventuais cálculos do colédoco, detectados durante a operação, nem sempre é feito pela via laparoscópica. Em função da falta de experiência ou de equipamentos, o encontro da coledocolitíase pode levar à associação de procedimentos endoscópicos ou conversão da cirurgia, modificando os resultados da colecistectomia videolaparoscópica.

A experiência nacional mostrada em estudo multicêntrico, ${ }^{14}$ revelou que, no tratamento da coledocolitíase (500 casos), em metade dos casos a resolução foi laparoscópica, em $20 \%$ optou-se pela papilotomia endoscópica préoperatória, em $17 \%$ pela papilotomia endoscópica pós-operatória e, em 14\%, pela conversão para a cirurgia aberta.

Um dos aspectos que chama a atenção é que à medida que o número das colecistectomias laparoscópicas aumentou, a indicação de procedimentos endoscópicos (papilotomia ou apenas colangiografias) também cresceu. A associação do tratamento endoscópico-laparoscópico passou a ser uma alternativa em relação à exploração convencional da via biliar e, por conseguinte, uma opção terapêutica na pancreatite biliar.

Embora tenha-se observado um aumento da indicação dos procedimentos endoscópicos-laparoscópicos, não se constatou aumento dos índices de morbidade e mortalidade. As taxas de sucesso terapêutico variam de $80 \%$ a $100 \%$, com morbidade de $6 \%$ a $9 \%$ e mortalidade inferior a $1 \% \cdot{ }^{15,16,17,18,19}$ No entanto, a realização sistemática da colangiografia no pré-operatório da colecistectomia laparoscópica revelou um número significativo de exames normais, da ordem de $50 \%$ a $60 \%$, de tal modo que os custos e riscos dessa indicação passaram a ser discutidos e a associação de procedimentos endoscópicos e laparoscópicos analisada. ${ }^{20,21,22}$

Se imaginarmos que no doente com pancreatite biliar a possibilidade de ocorrência da coledocolitíase possa 
ser maior, especialmente nos doentes submetidos a intervenções mais precoces, será que estaria justificando a realização sistemática da colangiografia endoscópica no préoperatório da colecistectomia laparoscópica? Não seria melhor indicar a colecistectomia laparoscópica postergada e realizar a colangiografia intra-operatória e, na eventualidade de se constatar cálculo no colédoco, tentar sua remoção pela mesma via? Quando estaria indicado tratar o doente por via endoscópica no pós-operatório? Quando converter a cirurgia para a via aberta? Essas questões permanecem sem uma resposta definitiva.

Soper et al, em 1994, ${ }^{23}$ relataram o emprego do tratamento endoscópico pré-operatório seguido da colecistectomia laparoscópica na pancreatite biliar em 23 doentes. Desses, somente em seis foi constatado cálculo no colédoco. Miller et al, em $1995^{24}$, realizaram o mesmo tipo de estudo em 11 casos e verificaram a presença do cálculo em apenas um doente. Ambos os trabalhos, apesar de mostrarem baixas morbidade e mortalidade na associação dos procedimentos, recomendaram o emprego da papilotomia endoscópica pré-operatória apenas em portadores de pancreatite biliar grave, em função da baixa incidência de coledocolitíase.

Em nossa casuística, indicamos a colangiografia endoscópica pré-operatória em 24 doentes $(22,42 \%)$, dos quais seis previamente à colecistectomia convencional e 18 à colecistectomia laparoscópica. Verificamos que o exame mostrou a presença de cálculo na via biliar em dez casos (34\%) e ausência do cálculo em 14 (56\%). Em um doente o exame mostrou apenas a presença de divertículo duodenal justapapilar. Mesmo nos doentes nos quais não se encontrou cálculo (14 casos), praticou-se a papilotomia endoscópica em oito (57\%). Em função dos baixos índices de coledocolitíase nos portadores de pancreatite biliar leve, a indicação sistemática da exploração endoscópica pré-operatória e da realização da papilotomia, mesmo não se encontrando cálculo, passou a ser discutível. O ideal seria a adoção de critérios clínicos, laboratoriais e de exames complementares que permitissem a seleção mais precisa de casos que se beneficiassem da associação desses procedimentos, evitando-se a papilotomia desnecessária.

No entanto, como os trabalhos que procuraram apontar os fatores de risco para a presença de cálculo na via biliar em portadores de pancreatite biliar, particularmente utilizando dados clínicos, dosagens de enzimas canali- culares e parâmetros ultra-sonográficos, não mostraram acurácia elevada, ${ }^{25,26,27}$ a colangiografia ainda é o método mais preciso para o diagnóstico. Assim, uma possibilidade seria seu emprego durante a colecistectomia laparoscópica postergada e, uma vez identificado o cálculo, a exploração da via biliar seria realizada.

$\mathrm{O}$ treinamento e a experiência com a via de acesso laparoscópica, associados ao desenvolvimento de instrumental e equipamentos, permitiram que alguns centros iniciassem a exploração do colédoco por essa via de acesso. As casuísticas iniciais ${ }^{28,29,30}$ mostraram a viabilidade do procedimento, tanto pela manipulação transcística, como pela coledocotomia. Em muitos casos, a remoção de cálculos tem sido feita com o auxílio de cateteres balonados ou pinças-cestas guiadas pela fluoroscopia, permitindo a remoção de cálculos.

Em nossa casuística, a comparação dos resultados entre as vias de acesso convencional e laparoscópica não mostrou diferenças importantes. Os intervalos de tempo de pré-operatório e a permanência hospitalar foram semelhantes. Apenas o tempo de pós-operatório foi diferente, isto é, os doentes operados pela via laparoscópica receberam alta hospitalar mais precoce. No entanto, devemos lembrar que nos doentes submetidos à cirurgia convencional, nem sempre praticou-se a colecistectomia com colangiografia. Operações de maior porte foram utilizadas (coledocotomia com drenagem à Kehr, papilotomia transduodenal e derivação biliodigestiva) implicando maior tempo de hospitalização.

Não observamos diferença estatística entre os índices de morbidade (14,1\% na convencional e 9,3\% na laparoscópica) e em ambos os grupos não houve mortalidade.

Em vista disso, consideramos que o doente portador de pancreatite leve deve ser submetido à operação postergada e que não há necessidade de se realizar sistematicamente a colangiografia endoscópica pré-operatória. O exame radiológico deve ser feito de rotina no intra-operatório, independente da via de acesso utilizada e, constatando-se a presença de cálculo, a exploração da via biliar para remoção do mesmo deve ser feita. Caso a via de acesso seja a laparoscópica, pode-se tentar a limpeza do colédoco por via trancística ou pela coledocotomia. Não havendo sucesso na remoção dos cálculos, deve-se considerar a conversão para a cirurgia aberta. Em casos selecionados, pode-se indicar o tratamento endoscópico pós-operatório, correndose o risco de uma reoperação, no insucesso do mesmo.

\begin{abstract}
A great deal of controversy still exists with regard to the surgical treatment of biliary pancreatitis. The question of the choice between an early intervention upon admission versus delayed operation performed during the same admission is still open to debate. The use of endoscopic procedures in any phase of the treatment, as well as new surgical approaches such as laparoscopic surgery show that the question on the most appropriate surgical treatment of biliary pancreatitis is far from it's end. In order to answer some of these questions, we retrospectively analized the early results of delayed conventional or laparoscopic operation in 107 patients with mild pancreatitis during a 9-year approach. Eighty patients were female and the mean age of the group was 46 years. Mean clinical manifestation time before arriving hospital the was 3,1 days, and after a mean hospitalization of 9,5 days the patients underwent surgical treatment. Intra-operative colangiography was performed in 102 patients and pre operative endoscopic colangiography in 24. The incidence of choledocolithiasis in this study was
\end{abstract}


$23 \%$, and morbidity was observed in $12 \%$. There was no mortality. In 64 patients conventional surgical treatment was employed while in 43 cases laparoscopic treatment was used. Results show significant statistic difference in the post operative hospital stay wich was shorter in the laparoscopic group. We concluded that a delayed surgical treatment for mild biliary pancretatitis has low morbidity and mortality, and can be performed by conventional or laparoscopic aproaches and choledocolithiasis did not increase morbidity or mortality.

Key Words: Acute Pancreatitis - Gallstone Pancreatitis - Surgical Treatment-Laparoscopic Cholecistectomy.

\section{REFERÊNCIAS}

1. Howard, J.M \& Jordan, G.L. Jr. - Relapsing pancreatitis secondary to choledocholithiasis . Arch. Sur., 73: 960-4, 1956.

2. Glenn, F. \& Frey, C.F. - Re-evaluation of the treatment of pancreatitis associated with biliary tract disease. Ann. Sur:; 1964, 160: 723-36.

3. Ranson, J.H.C - The timing of biliary surgery in acute pancreatitis. Ann. Surg.; 1979, 189: 654-63.

4. Frey, C.F. - The operative treatment of pancreatitis. Arch. Surg.; 1979, 98: 406-17.

5. Kelly, T.R., - Gallstone pancreatitis. Arch. Surg.; 1974, 109: 294-7

6. Acosta, J. M.: Rossi, R.: Galli, O.M.R. et al - Early surgery for acute gallstone pancreatitis evolution of a systematic approach. Surgery; 1978, 83: 367-70.

7. Safrany, L. \& Cotton, P. B. - A preliminary report: Urgent duodenoscopic sphincterotomy for acute gallstone pancreatitis. Surgery; 1981, 89: 424-8.

8. Neoptolemos, J.P.; London, N.: Slater, N. D.: Carrlocke, D.L. et al - A prospective study of ERCP and endoscopic sphincterotomy in the diagnosis and treatment of gallstone acute pancreatitis. Arch. Surg.; 1986, 121: 697-702.

9. Burch, J. M.; Feliciano, D.V.; Mattox, K. L et al - Gallstone pancreatitis: the question of time. Arch. Surg.; 1990, 125: 853-60.

10. Kowes, I.; Assef. J.C., Pacheco Junior, A.M. et al - Pancreatite aguda de origem biliar tratada cirurgicamente. G.E.D., 1987, 6: 17-22.

11. Kelly, T.R. \& Wagner, D.S. - Gallstone pancreatitis: a prospective randomized trial of the timing of surgery . Surgery; 1988, 104: 600-5..

12. Kim, U.; Sheth, M. - Optimal timing of surgical intervention in patients with acute pancreatitis associated with choletithiasis. Surg. Gynecol. Obstet.; 1980, 150: 499-501.

13. Schwesinger, W.H.; Page, C.P.; Siminek, K.R. et al - Biliary pancreratitis. Operative outcome with a selective approach. Arch. Surg.; 1991, 126:836-40.

14. Savassi-Rocha, P.R.; Ferreira, J.T.; Diniz, M.T.C. - Resultados da colecistectomia laparoscópica no Brasil: análise de 10.044 casos. In: DePaula, A.L.; Hashiba, K.; Bafutto, M. - Cirurgia Videolaparoscópica, Goiânia, Brasil, Gráfica e Editora Bandeirante, 1994. p. 201-7.

15. Cotton, P.B. - Endoscopic retrograd cholangiopancreatography and laparoscopic cholecystectomy. Am. J. Surg; 1993, 165: 474-8

16. Voyles, C.R. \& Sanders, D.L. - Common bile duct evaluation in the era of laparoscopic chole cystectomy. Ann. Surg.; 1994, 219: 744-52.

17. Duesing, R.A.; Williams, R.A.; Collins, J.C. et al - Managing Choledocholithiasis in the laparoscopic era. Am. J. Surg.; 1995, 170: 619-23.

18. Figueira, A. - Tratamento cirúrgico da colelitíase com coledocolitíase com e sem remoção endoscópica prévia dos cálculos do colédoco. São Paulo, 1995. ( Tese de Docência Livre - Universidade Federal de São Paulo - Escola Paulista de Medicina).
19. Arregui, M.E.; Davis, C.J.; Arkush, A.M. et al - Laparoscopic cholecystectomy combined with endoscopic sphyncterotomy and stone extraction or laparoscopic choledochoscopy and electrohydrautic lithotripsy for management of cholelithiasis with choledocholithiasis. Surg. Endosc.; 1992, 6: 10-5.

20. Graham, S.M.; Flowers, J.L.; Scott, T.R. et al - Laparoscopic cholecystectomy and common bile duct stones: The utility of planned perioperative endoscopic retrograde cholangiography and sphincterotomy-experience with 63 patients. Ann. Surg.; 1993, 218:61-7.

21. Schirmer, B.D. \& Dix, J. - Cost effectivenesess of laparoscopic cholecystectomy. J. Laparoendosc. Surg.; 1993, 2:79-83.

22. Berci, G. - Preoperative ERCP and intraoperative cholangiography in the age of laparoscopic cholecystectomy. Surg. Endosc.; 1993, 7:2.

23. Soper, N.J; Brent, M.; Callery, M.P. et al - Role of laparoscopic cholecystectomy in the management of acute gallstone pancreatitis. Am. J. Surg., 1994, 167:42-51.

24. Miller, R.E.; Kimmelstiel, F.M.; Winkler, W.P. - Management of common bile duct stones in the era of laparoscopic cholecystectomy. Am. J. Surg., 169:273-6, 1995.

25. McKay, A.J.; Duncan, J.G.; Imrie, C.W. - A prospective study of the clinical value and accuracy of grey scale ultrasound in detecting gallstones. Br. J. Surg.; 1978, 5: 330-3.

26. Dougherty, S.H.; Saltzstein, E.C.; Peacock, J.B. et al - Rapid resolution of high level hyperamylasemia as a guide to clinical diagnosis and timing of surgical treatment in patients with gallstones. Surg. Gynecol. Obstet., 1988, 166: 491-6.

27. Oria, A.; Alvarez, J.; Chiappetta, L. - Choledocholithiasis in acute gallstone pancreatitis: incidence and clinical significance. Arch. Surg., 1991, 126: 566-8.

28. Jacobs, M.; Verdeja, J.C.; Goldstein, H.S. - Laparoscopic choledochotomy. J. Laparoendosc. Surg.; 1991, 1: 79-82.

29. Hunter, J.G. - Laparoscopic trancystic common bile duct exploration. Am. J. Surg.; 1992, 163: 53-8.

30. DePaula, A.L.; Hashiba, K.; Bafutto, M. et al - Tratamento laparoscópico da coledocolitíase. In: DePaula, A.L.; Hashiba, K.; Bafutto, M. - Cirurgia Videolaparoscópica, Goiânia, Editora Bandeirante, 1993. p. 119-24.

\section{ENDEREÇO PARA CORRESPONDÊNCIA}

Dr. Rodrigo Altenfelder Silva

Rua Francisco Leitão, 469 - cj. 1.203

05414-010 - São Paulo-SP

Tel./Fax: (11) 853-0943 